\title{
HOMOTOPY LIMITS FOR 2-CATEGORIES
}

\author{
NICOLA GAMBINO
}

\begin{abstract}
We study homotopy limits for 2-categories using the theory of Quillen model categories. In order to do so, we establish the existence of projective and injective model structures on diagram 2-categories. Using these results, we describe the homotopical behaviour not only of conical limits but also of weighted limits. Finally, pseudo-limits are related to homotopy limits.
\end{abstract}

\section{QUILlEN MODEL STRUCTURES IN 2-CATEGORY THEORY}

The 2-category of small groupoids, functors, and natural transformations admits a model structure in which the weak equivalences are the equivalences of categories and the fibrations are the Grothendieck fibrations [1, 15]. Similarly, the 2-category of small categories, functors, and natural transformations admits a model structure in which the weak equivalences are the equivalences of categories and the fibrations are the isofibrations, which are functors satisfying a restricted version of the lifting condition for Grothendieck fibrations [15, 24]. Lack has vastly generalised these results by showing that every 2 -category $\mathcal{K}$ with finite limits and colimits admits a model structure, called here the natural model structure on $\mathcal{K}$, in which the weak equivalences are the equivalences in $\mathcal{K}$ and the fibrations are the isofibrations in $\mathcal{K}$ [21]. Here, the notions of equivalence and isofibration for a map in a 2-category are obtained by suitably generalising the notions of equivalence and of isofibration for a functor. We take Lack's theorem as a starting point to study homotopy limits for 2-categories.

Our first step is to show that for every small 2-category $\mathcal{A}$ and every 2-category $\mathcal{K}$ with finite limits and small colimits, the functor 2-category $[\mathcal{A}, \mathcal{K}]$ admits a model structure in which the weak equivalences are the pointwise equivalences and the fibrations are the pointwise isofibrations. We refer to this model structure as the projective model structure. When $\mathcal{K}$ is assumed to be locally presentable, the existence of the projective model structure follows by a result on the lifting of the natural model structure on a 2-category $\mathcal{K}$ to 2 -categories of algebras for a 2 -monad with rank on $\mathcal{K}[21$, Theorem 4.5]. However, the special form of the 2-category $[\mathcal{A}, \mathcal{K}]$ allows us to avoid assuming that $\mathcal{K}$ is locally presentable, and to give a proof of the model category axioms for the projective model structure which does not make any direct use of transfinite induction and provides explicit methods to produce the required factorisations and liftings.

We observe that by duality every 2 -category $\mathcal{K}$ with finite limits and colimits admits a dual of its natural model structure, in which the weak equivalences are the categorical equivalences and the cofibrations are the isocofibrations, that is to say the maps which are isofibrations in the 2 -category $\mathcal{K}^{\mathrm{op}}$, obtained by reversing maps, but not 2 -cells, of $\mathcal{K}$. We will then show that every small 2-category $\mathcal{A}$ and every 2-category $\mathcal{K}$ with small limits and finite colimits, the functor 2-category $[\mathcal{A}, \mathcal{K}]$ admits a model structure in which the weak equivalences are the pointwise equivalences and the cofibrations are the pointwise isocofibrations. We refer to this model structure as the injective model structure. 
The existence of the projective and injective model structures allows us to apply the general theory of enriched model categories $[9,14,25]$ to study the total derived functors of limit 2 -functors. We will consider not only conical limits but also weighted limits $[17,18,27]$. The study of homotopy-theoretic aspects of weighted limits for 2-categories reveals that there are two different combinations of model structures on 2-categories of diagrams that allow us to regard the weighted limit 2-functor as a right Quillen 2-functor in two variables. This observation gives rise to two different, but equivalent, ways of describing weighted homotopy limits in terms of weighed limits.

In order to describe precisely the completeness properties of many 2-categories of interest, such as those of categories equipped with algebraic structure [4], there is a rich theory of 2-categorical limits $[3,18,23,27]$. We will relate homotopy limits to pseudo-limits $[18,27]$. We do so not only at the level of universal properties, but also by showing how the two reductions of weighted homotopy limits to weighted limits correspond to two ways of reducing the weighted pseudo-limits to weighted limits. These results provide a precise connection between ideas on homotopical coherence and on categorical coherence, confirming the idea, implicit in much of the development of higher-dimensional category theory, that homotopical and categorical coherence should be viewed as two aspects of the same phenomenon.

Let us point out that the approach of using model categories to study homotopy limits in terms of derived functors, which goes back to [5] (see [13] for a modern treatment), is not the only possible one. A different approach, based on the notion of homotopical category, is developed in [7]. The paper [26] provides a discussion of the relationship between these approaches and the one involving the bar construction [22]. Yet another approach is developed in [6].

Remark. For the convenience of the reader, the axioms for a Quillen model category are recalled in Appendix A.

\section{Model 2-CATEgORIES}

2.1. The natural model structure on Cat. As a special case of $[15$, Theorem 4], the category Cat of small categories and functors admits a model structure in which the weak equivalences are the fully faithful and essentially surjective functors and the cofibrations are the functors injective on objects. Using the Axiom of Choice, which is not necessary to establish this result, the weak equivalences can be identified with the categorical equivalences, that is to say functors $f: A \rightarrow B$ such that there exists a functor $g: B \rightarrow A$ and natural isomorphisms $\eta: 1_{A} \Rightarrow g f$ and $\varepsilon: f g \Rightarrow 1_{B}$, and the fibrations with the isofibrations, that is to say functors $f: A \rightarrow B$ such that for every $a \in A, b \in B$ and isomorphism $\beta: f a \rightarrow b$, there exists a lifting of $\beta$, given by an $a^{\prime} \in A$ and an isomorphism $\alpha: a \rightarrow a^{\prime}$ such that $f a^{\prime}=b$ and $f \alpha=\beta$. We refer to this model structure as the natural model structure on Cat. We write $\mathrm{Ho}$ (Cat) for the homotopy category of Cat, which consists of categories and isomorphism classes of functors, and denote the localization functor as $\lambda$ : Cat $\rightarrow \mathrm{Ho}(\mathbf{C a t})$. Let us point out that there is a different model structure on Cat, originally established by Thomason [28], which is not going to be considered here.

2.2. Model 2-categories. The cartesian product equips Cat with a symmetric monoidal structure which, as recalled in $[21, \S 2.2]$, satisfies the axioms for a monoidal model category $[9,14,25]$. For any symmetric monoidal model category $\mathcal{V}$, there is an associated notion of a model $\mathcal{V}$-enriched category $[9,14]$. We spell out the general definition in the special case when $\mathcal{V}$ is Cat, so as to obtain the notion of a model 2-category. In order to do so, for a 2-category $\mathcal{K}$, let us write $\mathcal{K}(X, Y)$ for the hom-category associated to a pair of objects $X, Y \in \mathcal{K}$. A pair of maps 
$u: X \rightarrow Y$ and $v: V \rightarrow W$ in $\mathcal{K}$ determines the commutative diagram of categories and functors

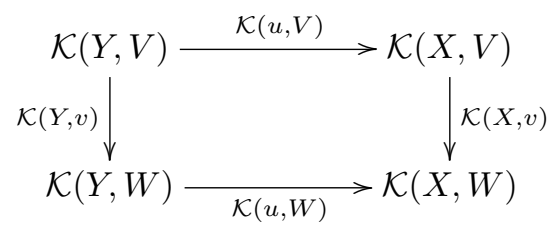

Since Cat has pullbacks, we obtain a canonical functor, denoted

$$
[u, v]: \mathcal{K}(Y, V) \rightarrow \mathcal{K}(Y, W) \times_{\mathcal{K}(X, W)} \mathcal{K}(X, V)
$$

This map is used in Definition 2.2.1.

Definition 2.2.1. Let $\mathcal{K}$ be a 2-category with finite limits and colimits. A model 2 -structure on $\mathcal{K}$ consists of a model structure on its underlying category such that the following condition holds: if $u: X \rightarrow Y$ is a cofibration and $v: V \rightarrow W$ is a fibration in $\mathcal{K}$, then the functor $[u, v]$ is an isofibration in Cat, which is a categorical equivalence whenever either $u$ or $v$ is a weak equivalence. A model 2-category is a 2category with finite limits and colimits which is equipped with a model 2 -structure.

For a 2 -category $\mathcal{K}$, we write $\mathcal{K}^{\text {op }}$ for the category obtained from $\mathcal{K}$ by formally reversing the direction of the maps of $\mathcal{K}$, but leaving the 2 -cells unchanged. The function mapping a pairs of objects $X, Y \in \mathcal{K}$ to the category $\mathcal{K}(X, Y)$ determines a 2-functor $\mathcal{K}(-,-): \mathcal{K}^{\text {op }} \times \mathcal{K} \rightarrow$ Cat. We say that $\mathcal{K}$ has tensors if for every $X \in \mathcal{K}$ the 2-functor $\mathcal{K}(X,-): \mathcal{K} \rightarrow$ Cat has a left 2-adjoint. The left 2-adjoint sends $A \in \mathbf{C a t}$ into $A \otimes X \in \mathcal{K}$, the $A$-tensor of $X$, and the 2-adjointness means that we have a 2-natural isomorphism with components

$$
\mathcal{K}(A \otimes X, Y) \cong \mathbf{C a t}(A, \mathcal{K}(X, Y)) .
$$

Similarly, we say that $\mathcal{K}$ has cotensors if for every $Y \in \mathcal{K}$, the functor $\mathcal{K}(-, Y)$ : $\mathcal{K}^{\text {op }} \rightarrow$ Cat has a left adjoint. The left 2-adjoint sends $A \in \mathbf{C a t}$ into $Y^{A} \in \mathcal{K}$, the $A$ cotensor of $Y$. Here, the 2-adjointness means that we have a 2-natural isomorphism with components

$$
\mathcal{K}\left(X, Y^{A}\right) \cong \mathbf{C a t}(A, \mathcal{K}(X, Y)) .
$$

It is convenient to have equivalent characterizations of the notion of a model 2category under the assumption that $\mathcal{K}$ is tensored or cotensored. This involves the construction of analogues of the map defined in (2). Let us consider maps $f: A \rightarrow B$ in Cat and $u: X \rightarrow Y$ in $\mathcal{K}$. When $\mathcal{K}$ has tensors, there is an evident analogue of the diagram in (1), and the universal property of pushouts gives us canonical map

$$
\langle f, u\rangle:(A \otimes Y) \sqcup_{A \otimes X}(B \otimes X) \rightarrow B \otimes Y .
$$

When $\mathcal{K}$ has cotensors, the universal property of pullbacks gives us a canonical map

$$
\{f, u\}: X^{B} \rightarrow Y^{B} \times_{Y^{A}} X^{A} .
$$

Lemma 2.2.2 is a special case of [9, Proposition 3.4]. It will be useful in the study of homotopy-theoretic aspects of 2-categorical limits.

Lemma 2.2.2. Let $\mathcal{K}$ be a 2 -category with finite limits whose underlying category is equipped with a model structure.

(i) If $\mathcal{K}$ is tensored, $\mathcal{K}$ is a model 2-category if and only if the following conditions hold: if $f$ is a cofibration in Cat and $u$ is a fibration in $\mathcal{K}$, then $\langle f, u\rangle$ is a cofibration in $\mathcal{K}$, which is a weak equivalence whenever either $f$ or $u$ is so.

(ii) If $\mathcal{K}$ is cotensored, $\mathcal{K}$ is a model 2 -category if and only if the following condition holds: if $f$ is a cofibration in Cat and $u$ is a fibration in $\mathcal{K}$, then $\{f, u\}$ is a fibration in $\mathcal{K}$ which is a weak equivalence whenever either $f$ or $u$ is so. 
While the definition of a model 2-category involves a compatibilty condition between different model structures, the notion of a Quillen 2-adjunction can be formulated in a straightforward fashion, simply recalling that a 2 -adjunction between 2-categories determines an adjunction between their underlying categories. A Quillen 2-adjunction between model 2-categories consists of a 2-adjunction whose underlying adjunction is a Quillen adjunction $[14, \S 1.3 .1]$. The notion of Quillen 2-equivalence is defined analgously, using the familiar notion of a Quillen equivalence $[14, \S 1.3 .3]$. The notion of a Quillen 2-functor in two variables is discussed in Section 5.1.

2.3. The natural model structure on a 2-category. The notions of categorical equivalence and of isofibrations recalled in Section 2.1 can be expressed not only in Cat, but within any 2-category $\mathcal{K}$ as follows. We call a map $f: B \rightarrow A$ in $\mathcal{K}$ an equivalence if there exists a map $g: A \rightarrow B$ and invertible 2-cells $\eta: 1_{A} \Rightarrow g f$ and $\varepsilon: f g \Rightarrow 1_{B}$. We refer to such $g: B \rightarrow A$ as a quasi-inverse of $f: A \rightarrow B$. If an equivalence has a section, then it is called a surjective equivalence; if it has a retraction then it is called an injective equivalence. For example, given diagrams of form
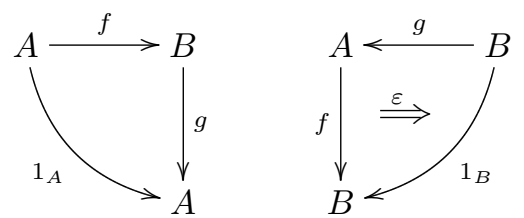

where $\varepsilon$ is an invertible 2-cell, then $f$ is an injective equivalence and $g$ is a surjective equivalence. Following $[21, \S 3.4]$, we define a map $f: A \rightarrow B$ to be an isofibration if for every diagram of form

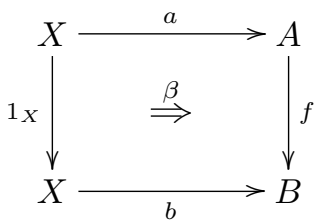

where $\beta: b \Rightarrow f a$ is an invertible 2 -cell, there exists a map $a^{\prime}: X \rightarrow A$ such that $b=f a^{\prime}$ and an invertible 2-cell $\alpha: a^{\prime} \Rightarrow a$ such that $\beta: b \Rightarrow f a$ equals the composite 2-cell $f \alpha: b \Rightarrow f a$, obtained by the following pasting diagram

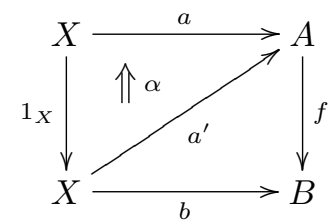

As shown in [21, Section 3] every 2-category $\mathcal{K}$ with finite limits and colimits admits a model 2-structure in which the weak equivalences are the equivalences and the fibrations are the isofibrations. We refer to this model structure as the natural model structure on $\mathcal{K}$. The acyclic fibrations in the natural model structure are the surjective equivalences. The special case of this result for the 2-category Cat of small categories, functors, and natural transformations gives back the natural model structure discussed in Section 2.1 and implies that it is a model 2-category structure.

\section{Projective model STRuCtures on 2-CATEgories of Diagrams}

3.1. Pointwise equivalences. For a small 2-category $\mathcal{A}$, we refer to 2 -functors $F: \mathcal{A} \rightarrow \mathcal{K}$ as diagrams. We write $[\mathcal{A}, \mathcal{K}]$ for the 2-category of diagrams, 2-natural 
transformations, and modifications [20]. This 2-category has again finite limits and colimits, since all limits and colimits are computed pointwise $[17,18]$. We say that a 2-natural transformation $m: F \rightarrow G$, where $F$ and $G$ are diagrams, is a pointwise equivalence if all its components $m_{A}: F A \rightarrow G A$ are equivalences in $\mathcal{K}$. In general, a pointwise equivalence is not an equivalence in $[\mathcal{A}, \mathcal{K}]$. However, as we recall below, the results in [16] imply that a pointwise equivalence is an equivalence in the larger 2-category $\operatorname{Psd}[\mathcal{A}, \mathcal{K}]$ of diagrams, pseudo-natural transformations, and modifications. Let us recall that the notion of a pseudo-natural transformation generalises that of a 2-natural transformations by allowing the 2-naturality squares to commute up to coherent isomorphism rather than strictly. More precisely, given diagrams $F$ and $G$, a pseudo-natural transformation $m: F \rightarrow G$ consists of a family of maps $m_{A}: F A \rightarrow G A$ in $\mathcal{K}$, for $A \in \mathcal{A}$, and a family of invertible 2-cells $m_{u}$, for $u: A \rightarrow B$ in $\mathcal{A}$, fitting in diagrams of form

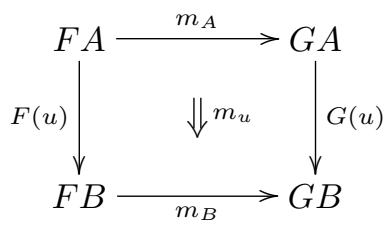

These 2-cells are subject to coherence axioms [27, §4] that express suitable compatibility conditions with respect to identity and composition of maps in $\mathcal{A}$, and with composition with 2 -cells in $\mathcal{A}$. A 2-natural transformation can be seen as a pseudo-natural transformation and therefore there is an inclusion 2-functor

$$
[\mathcal{A}, \mathcal{K}] \stackrel{I}{\longrightarrow} \operatorname{Psd}[\mathcal{A}, \mathcal{K}] .
$$

If $m: F \rightarrow G$ is a a pointwise equivalence, then there exists a pseudo-natural transformation $n: G \rightarrow F$ that is a pointwise quasi-inverse for $m: F \rightarrow G$. Indeed, for $A \in \mathcal{A}$, let $n_{A}: G A \rightarrow F A$ be a quasi-inverse to $m_{A}: F A \rightarrow G A$ in $\mathcal{K}$. We can assume without loss of generality that $m_{A}$ and $n_{A}$ form an adjoint equivalence in $\mathcal{K}$,

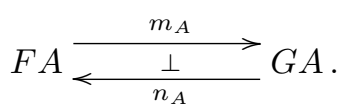

Let us write $\eta_{A}: 1_{F A} \Rightarrow n_{A} m_{A}$ and $\varepsilon_{A}: m_{A} n_{A} \Rightarrow 1_{G A}$ for the invertible 2-cells providing the unit and counit of the adjoint equivalence, respectively. Using them, we can equip the family $n_{A}: G A \rightarrow F A$ with 2 -cells so as to obtain a pseudonatural transformation $n: G \rightarrow F$. For $u: A \rightarrow B$ in $\mathcal{A}$, we define a 2-cell $n_{u}: F(u) n_{A} \Rightarrow n_{B} G(u)$ as the composite of the invertible 2-cells appearing in the following diagram:

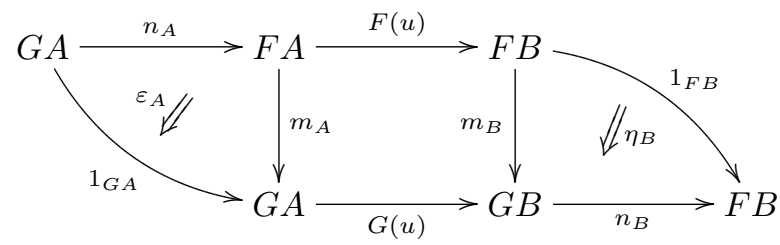

With this definition, the coherence axioms for a pseudo-natural transformations follow from the triangular laws of the adjoint equivalence in (9).

3.2. The projective model structure. Let $\mathcal{A}$ be a small category, and let $\mathcal{K}$ be a model 2-category. A 2-natural transformation $m: F \rightarrow G$ between diagrams is said to be a pointwise weak equivalence if all of its components $m_{A}: F A \rightarrow G A$ are weak equivalences in the model 2 -structure on $\mathcal{K}$. The notions of pointwise fibration and 
pointwise cofibration are defined analogously. We say that a 2-natural transformation is a projective cofibration if it has the left lifting property with respect to the 2-natural transformations which are pointwise acyclic fibrations. Relative to the model 2-category structure on $\mathcal{K}$, the projective model structure on the 2-category $[\mathcal{A}, \mathcal{K}]$ is defined as follows:

$$
[\mathcal{A}, \mathcal{K}]_{\text {Proj }}= \begin{cases}\text { Weak equivalences } & =\text { pointwise weak equivalences } \\ \text { Fibrations } & =\text { pointwise fibrations } \\ \text { Cofibrations } & =\text { projective cofibrations. }\end{cases}
$$

When the model structure $\mathcal{K}$ is cofibrantly generated, the existence of the projective model structure is a familiar fact [13]. In general, however, it is not known whether these definitions satisfy Quillen's axioms for a model category.

Let us now consider a 2 -category $\mathcal{K}$ with finite limits and colimits, and regard it as equipped with its natural model 2-structure. This model structure is not, in general, cofibrantly generated [21, Proposition 3.19]. We will show that if $\mathcal{K}$ is cocomplete, then the diagram 2 -category $[\mathcal{A}, \mathcal{K}]$ admits the projective model structure. Let us emphasize that the projective model structure we will establish is relative to the natural model structure on $\mathcal{K}$. Therefore, a pointwise weak equivalence is a pointwise equivalence, a pointwise fibration is a pointwise isofibration, and so a pointwise acyclic fibration is a pointwise surjective equivalence. The proof of Lemma 3.2.1 uses the observations on pseudo-natural transformations made in Section 3.1.

Lemma 3.2.1. Let $m: F \rightarrow G$ be a 2-natural transformation that is a pointwise cofibration, and let $n: H \rightarrow K$ be a 2-natural transformation that is a pointwise fibration. If either $m$ or $n$ is a pointwise weak equivalence, then for every commutative square in $\operatorname{Psd}[\mathcal{A}, \mathcal{K}]$ of the form

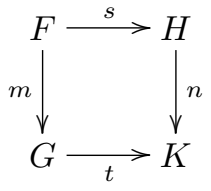

there exists a pseudo-natural transformation $j: G \rightarrow H$ that is a filler for the diagram.

Proof. Let $m: F \rightarrow G$ be a pointwise equivalence, and so a pointwise acyclic cofibration. Since $n: H \rightarrow K$ is a pointwise fibration, for every $A \in \mathcal{A}$ there exists a filler for the commutative diagram

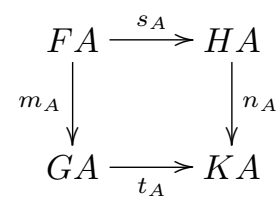

Let $j_{A}: G A \rightarrow H A$ be a filler. Since $m: F \rightarrow G$ is a pointwise equivalence, we can use its pseudo-natural quasi-inverse to construct 2-cells making the maps $j_{A}: G A \rightarrow H A$ into a pseudo-natural transformation. The case when $n: H \rightarrow K$ is a pointwise acyclic fibration is treated analogously.

Lemma 3.2.2. Every 2-natural transformation can be factored both as a pointwise cofibration followed by a pointwise acyclic fibration, and as a pointwise acyclic cofibration followed by a pointwise fibration.

Proof. The factorisations in $\mathcal{K}$ are functorial.

We now assume that $\mathcal{K}$ is also cocomplete, so as to be able to apply the results in [4]. Recall that we write $\operatorname{Psd}[\mathcal{A}, \mathcal{K}]$ for the 2-category of diagrams, pseudo-natural 
transformations, and modifications. A crucial fact underlying our verification of Quillen's axioms for the projective model structure on $[\mathcal{A}, \mathcal{K}]$ is the consequence of $[4$, Theorem 3.16] exhibiting a 2-adjunction of form

$$
[\mathcal{A}, \mathcal{K}] \underset{\frac{(-)^{\prime}}{I}}{\frac{\perp}{I}} \operatorname{Psd}[\mathcal{A}, \mathcal{K}] .
$$

The right 2 -adjoint $I$ is the inclusion 2 -functor in (8). Since $I$ is an inclusion, it will not be mentioned explicitly in the following. The left 2 -adjoint maps a diagram $F$ into a diagram $F^{\prime}$, called the flexible diagram associated to $F$. The components of the unit of the 2-adjunction are pseudo-natural transformations $p_{F}: F \rightarrow F^{\prime}$ which are universal in the sense that every pseudo-natural transformation $m: F \rightarrow G$ factors uniquely through $p_{F}: F \rightarrow F^{\prime}$ in a diagram of form

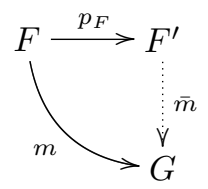

The components of the counit are 2-natural transformations $q_{F}: F^{\prime} \rightarrow F$. As shown within the theory of 2-monads in [4, Theorem 4.2] and explained in the special case of interest to us in $[3, \S 4]$, the pseudo-natural transformations $p_{F}$ and the 2-natural transformations $q_{F}$ form an adjoint equivalence. In particular, we have diagrams

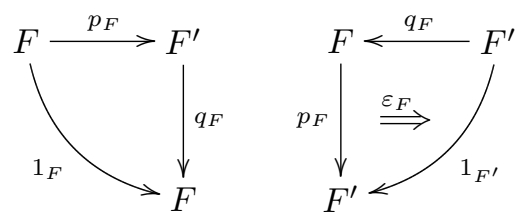

The 2-cell $\varepsilon_{F}: p_{F} q_{F} \Rightarrow 1_{F^{\prime}}$ is the invertible modification providing the counit of the adjoint equivalence. Lemma 3.2.3 states an important property of the counit of the 2-adjunction in (10). As we will see, the flexible 2-functor associated to a 2 -functor can be seen as its cofibrant replacement with respect to the projective model structure on $[\mathcal{A}, \mathcal{K}]$.

Lemma 3.2.3. For every $F$, the 2-natural transformation $q_{F}: F^{\prime} \rightarrow F$ is a pointwise acyclic fibration.

Proof. We need to show that $q_{F}: F^{\prime} \rightarrow F$ is a pointwise surjective equivalence in $\mathcal{K}$. This follows by instanciating pointwise the diagrams in (11).

For the statement of Lemma 3.2.4, recall that a 2-natural transformation is a projective cofibration if it has the left lifting property with respect to the pointwise acyclic fibrations. We also say that a 2-natural transformation is a projective trivial cofibration if it has the left lifting property with respect to the pointwise fibrations.

Lemma 3.2.4. If $m: F \rightarrow G$ is a pointwise cofibration, then $m^{\prime}: F^{\prime} \rightarrow G^{\prime}$ is a projective cofibration. Furthermore, when $m$ is a pointwise acyclic cofibration, then $m^{\prime}$ is a projective trivial cofibration.

Proof. Assume that $m$ is a pointwise cofibration. It is necessary to show that $m^{\prime}$ has the left lifting property with respect to pointwise acyclic fibrations. In order to do so, first use the 2-adjunction in (10) to transfer the lifting problem from $[\mathcal{A}, \mathcal{K}]$ to $\operatorname{Psd}[\mathcal{A}, \mathcal{K}]$, and then apply Lemma 3.2.1. The proof of the other claim is analogous. 
Lemma 3.2.5 is the crucial step to establish that the projective model structure satisfies the axioms for a Quillen model structure. In its proof, we use again the 2 -adjunction in (10).

Lemma 3.2.5. Every pointwise cofibration $m: F \rightarrow G$ can be factored as follows

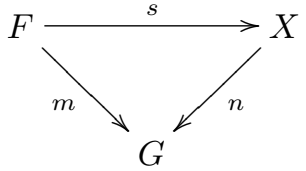

where $s: F \rightarrow X$ is a projective cofibration, and $n: X \rightarrow G$ is a pointwise acyclic fibration. Furthermore, if $m: F \rightarrow G$ is a pointwise weak equivalence, and so a pointwise acyclic cofibration, then $s: F \rightarrow X$ is a projective trivial cofibration.

Proof. First, recall that by Lemma 3.2.4, $m^{\prime}: F^{\prime} \rightarrow G^{\prime}$ is a projective cofibration. To construct the required factorisation, we use the 2-naturality of the counit of the 2-adjoint in (10). We define $s: F \rightarrow X$ as the pushout of $m^{\prime}: F^{\prime} \rightarrow G^{\prime}$ along $q_{F}: F^{\prime} \rightarrow F$. The 2-naturality of the counit determines a canonical 2-natural transformation $n: X \rightarrow G$ fitting in the following diagram:

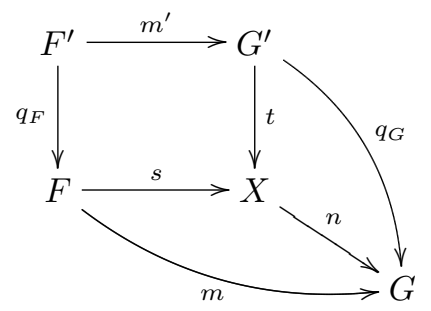

Recall that, being defined as the maps having the left lifting property with respect to the pointwise acyclic fibrations, projective cofibrations are closed under pushouts. Therefore $s: F \rightarrow X$ is a projective cofibration, since it is the pushout of $m^{\prime}: F^{\prime} \rightarrow$ $G^{\prime}$, which is a projective cofibration.

To show that $n: X \rightarrow G$ is a pointwise acyclic fibration, we begin by showing that it is a pointwise weak equivalence. In order to do this, we want to apply pointwise the Three-for-Two Axiom to the commuting triangle involving $q_{G}: G^{\prime} \rightarrow G$ and $t: G^{\prime} \rightarrow X$. We have already seen that $q_{G}$ is a pointwise weak equivalence. For $A \in \mathcal{A}$, to show that $t_{A}: G^{\prime} A \rightarrow X A$ is a weak equivalence, observe that it is the pushout of $q_{F A}$ along $m_{A}^{\prime}$, since pushouts in $[\mathcal{A}, \mathcal{K}]$ are also computed pointwise. But $q_{F A}: F^{\prime} A \rightarrow F A$ is a weak equivalence and $m_{A}^{\prime}: F^{\prime} A \rightarrow G^{\prime} A$ is a cofibration, since every projective cofibration is also a pointwise cofibration. Since every object in $\mathcal{K}$ is cofibrant, we can apply a result of Reedy [13, Proposition 13.1.2] and deduce that $t_{A}$, being the pushout of a weak equivalence between cofibrant objects along a cofibration, is a weak equivalence.

Finally, we need to show that $n: X \rightarrow G$ is a pointwise acyclic fibration, which amounts to showing that it is a pointwise surjective equivalence. This follows by the commutativity of triangle involving $n$ and $q_{G}$ in (12), since $q_{G}: G^{\prime} \rightarrow G$ is a pointwise surjective equivalence, as shown in Lemma 3.2.3.

The second claim follows from the construction given above. First, observe that if $m: F \rightarrow G$ is a pointwise acyclic cofibration, then $m^{\prime}: F^{\prime} \rightarrow G^{\prime}$ is a projective trivial cofibration by Lemma 3.2.4. Since $s: F \rightarrow X$ is a pushout of $m^{\prime}: F^{\prime} \rightarrow G^{\prime}$, it inherits from $m^{\prime}$ the left lifting property with respect to the pointwise fibrations.

We can now prove the existence of the projective model structure. The axioms for a Quillen model category, Three-For-Two (Q1), Retracts (Q2), Lifting (Q3), and Factorisations (Q4) are recalled in Appendix A. We also prove that it satisfies the additional axiom of a model 2-category, recalled in Definition 2.2.1. 
Theorem 3.2.6. Let $\mathcal{K}$ be a 2-category with finite limits and colimits, considered as equipped with its natural model 2 -category structure. If $\mathcal{K}$ is cocomplete, then for every small 2-category $\mathcal{A}$ the 2 -category $[\mathcal{A}, \mathcal{K}]$ admits the projective model structure. The projective model structure equips $[\mathcal{A}, \mathcal{K}]$ with a model 2 -category structure.

Proof. The verification of (Q1) and (Q2) is straightforward. For the rest of the proof, let us refer to a map that is both a projective cofibration and a pointwise weak equivalence as a projective acyclic cofibration. Also, recall that a projective trivial cofibration is a 2-natural transformation with the left lifting property with respect to pointwise acyclic fibrations.

We verify (Q4), which involves providing two factorisations. Suppose we wish to factor $m: F \rightarrow G$ as a projective cofibration followed by a pointwise acyclic fibration. First, apply Lemma 3.2 .2 so as to factor $m$ as a pointwise cofibration followed by a pointwise acyclic fibration. Secondly, apply Lemma 3.2.5 and factor the pointwise cofibration just obtained as a projective cofibration followed by a pointwise acyclic fibration. The projective cofibration is the first component of the required factorisation, while the second is given by a composition of pointwise acyclic fibrations, which is an acyclic fibration. Next, suppose we wish to factor $m: F \rightarrow G$ as a projective acyclic cofibration followed by a pointwise fibration. First, apply Lemma 3.2.2 and factor $m$ as a pointwise acyclic cofibration followed by a pointwise fibration. Secondly, apply Lemma 3.2.5 and factor the pointwise acyclic cofibration as a projective cofibration followed by a pointwise acyclic fibration. Now, observe that the projective cofibration is in fact a pointwise equivalence by Three-For-Two, and hence it is a projective acyclic cofibration. This provides the first part of the required factorisation. The second part is the composite of a pointwise fibration followed by a pointwise acyclic fibration, and hence it is a pointwise fibration, as required.

Finally, we prove that (Q3) holds. The first part of the statement follows by the very definition of projective cofibration. For the second part, it suffices to verify that a projective cofibration $m: F \rightarrow G$ is a pointwise weak equivalence if and only if it is a projective trivial cofibration. Let $m: F \rightarrow G$ be a projective cofibration. First, we assume that it is a pointwise weak equivalence. By Lemma 3.2.5 we can factor $m$ as a projective trivial cofibration followed by a pointwise acyclic fibration. Next, we use the lifting property of projective cofibrations with respect to pointwise acyclic fibrations to exhibit $m$ as a retract of the projective trivial cofibration that we obtained in the factorisation, which implies that $m$ is a projective trivial cofibration as well. For the converse implication, let $m: F \rightarrow G$ be a projective trivial cofibration. Then, we can factor it as a pointwise cofibration followed by a pointwise acyclic fibration. The lifting property of projective trivial cofibrations with respect to pointwise acyclic fibrations shows that $m$ is a retract of the pointwise acyclic cofibration with which we factored it, and hence it is a pointwise weak equivalence, as required.

To check that the projective model structure is a model 2-category structure, we use part $(i i)$ of Lemma 2.2.2. Since cotensors in diagram 2-categories are computed pointwise, the required statement follows from the corresponding fact for $\mathcal{K}$.

As an instance of the general definition of flexible algebra for a 2-monad [4], a diagram $F$ is said to be flexible if $q_{F}: F^{\prime} \rightarrow F$ has a section in $[\mathcal{A}, \mathcal{K}]$. By the same argument used in the proof of [21, Theorem 4.12] we obtain that $F$ is flexible if and only if it is projectively cofibrant. The 2-adjunction in (10) determines a 2 -comonad on $[\mathcal{A}, \mathcal{K}]$ whose underlying 2-functor $Q:[\mathcal{A}, \mathcal{K}] \rightarrow[\mathcal{A}, \mathcal{K}]$ is defined by letting $Q F=(I F)^{\prime}$. The counit of the 2-adjunction is the counit of the 2-comonad, and so we can write its components as $q_{F}: Q F \rightarrow F$. Since every 2-functor of the form $Q F$ is projectively cofibrant, the acyclic fibrations $q_{F}: Q F \rightarrow F$ provide 2 -functorial cofibrant replacements for the projective model structure. Comonadic 
cofibrant replacements of this type are studied in [8]. It is immediate to see instead that every diagram is projectively fibrant.

\section{INJECTIVE MODEL STRUCTURES ON 2-CATEGORIES OF DIAGRAMS}

4.1. The dual of the natural model structure on a 2-category. The notion of equivalence being self-dual, a map is an equivalence in $\mathcal{K}$ if and only if it is an equivalence in $\mathcal{K}^{\mathrm{op}}$. We say that a map is an isocofibration in $\mathcal{K}$ if it is an isofibration in $\mathcal{K}^{\text {op }}$. By duality, we obtain the following result.

Proposition 4.1.1. Every 2-category $\mathcal{K}$ with finite limits and colimits admits a model 2-structure in which the weak equivalences are the equivalences and the cofibrations are the isocofibrations.

Proof. The natural model structure on the 2-category $\mathcal{K}^{\text {op }}$ determines a model structrure on $\left(\mathcal{K}^{\mathrm{op}}\right)^{\text {op }}$ whose weak equivalences are the weak equivalences in the natural model structure on $\mathcal{K}^{\mathrm{op}}$ and whose cofibrations are the fibrations in the natural model structure on $\mathcal{K}^{\mathrm{op}}$. Since $\left(\mathcal{K}^{\mathrm{op}}\right)^{\text {op }}$ is nothing but $\mathcal{K}$, we obtain the required model structure on $\mathcal{K}$. The fact that it is a model 2 -structure follows easily from the fact that the natural model structure is so.

We refer to the model structure of Proposition 4.1.1 as the dual of the natural model structure on $\mathcal{K}$. The acyclic cofibrations in the dual of the model structure are the injective equivalences. We wish to relate the natural model structure on $\mathcal{K}$ and its dual. Let us write $J$ for the category with two objects and an isomorphism between them. We can identify functors $J \rightarrow \mathcal{K}(X, B)$ with the data of a pair of maps $b_{0}: X \rightarrow B, b_{1}: X \rightarrow B$ and an invertible 2 -cell $\beta: b_{0} \rightarrow b_{1}$ in $\mathcal{K}$. For $X \in \mathcal{K}$, the universal property of tensors in (3) implies that the $J$-tensor of $X$, written $J \otimes X$, comes equipped with a canonical functor $e: J \rightarrow \mathcal{K}(X, J \otimes X)$ which induces by composition a natural isomorphism of categories with components $\mathcal{K}(J \otimes X, B) \cong$ $\operatorname{Cat}(J, \mathcal{K}(X, B))$. The map $e_{0}: X \rightarrow J \otimes X$ is an injective equivalence, and hence an acyclic cofibration in the dual of the natural model structure on $\mathcal{K}$.

Lemma 4.1.2. Let $\mathcal{K}$ be a 2-category with finite limits and colimits.

(i) If a map has the right lifting property with respect to the injective equivalences, then it is an isofibration.

(ii) If a map has the left lifting property with respect to the surjective equivalences, then it is an isocofibration.

Proof. First, observe that $(i)$ and $(i i)$ are equivalent by duality. We prove $(i)$. Let $f: A \rightarrow B$ be a map with the right lifting property with respect to injective equivalences. Given a diagram as in (5), the 2-cell $\beta: b \Rightarrow f a$ induces a functor $J \rightarrow \mathcal{K}(X, B)$. By the universal property of $J \otimes X$, we obtain a map $\langle\beta\rangle: J \otimes X \rightarrow B$ making the following diagram commute

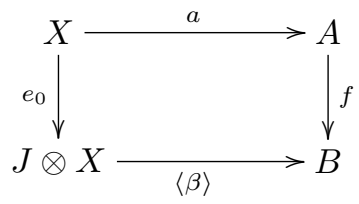

Since $f: A \rightarrow B$ has the right lifting property with respect to injective equivalences and $e_{0}: X \rightarrow J \otimes X$ is an injective equivalence, there is a filler for the diagram, which can be used to construct the appropriate 1-cells and 2-cells for the diagram in (6), thus showing that $f: A \rightarrow B$ is an isofibration, as required.

Proposition 4.1.3. Let $\mathcal{K}$ be a 2-category with finite limits and colimits. The natural model structure on $\mathcal{K}$ and its dual are Quillen 2-equivalent. 
Proof. We consider the identity 2-functor as going from $\mathcal{K}$ equipped with its natural model structure to $\mathcal{K}$ equipped with the dual of the natural model structure. We wish to show that it is left Quillen 2-functor. The identity 2-functor clearly preserves weak equivalences. Since the cofibrations in the natural model structure are the maps with the left lifting property with respect to the surjective equivalences, and the cofibrations in the dual of the natural model structure are the isocofibrations, part (ii) of Lemma 4.1.2 shows that the identity preserves cofibrations. Hence the identity 2-functor preserves weak equivalences and acyclic cofibrations, as required.

The natural model structure on Cat and its dual not only are Quillen 2-equivalent, but actually coincide.

4.2. The injective model structure. Let $\mathcal{A}$ be a small 2 -category and $\mathcal{K}$ be a model 2-category. A 2-natural transformation between diagrams is said to be an injective fibration if it has the right lifting property with respect to the pointwise acyclic cofibrations. Relative to the model 2-structure on $\mathcal{K}$, we define the injective model structure on $[\mathcal{A}, \mathcal{K}]$ as follows:

$$
[\mathcal{A}, \mathcal{K}]_{\text {Inj }}= \begin{cases}\text { Weak equivalences } & =\text { pointwise weak equivalences } \\ \text { Fibrations } & =\text { injective fibrations } \\ \text { Cofibrations } & =\text { pointwise cofibrations. }\end{cases}
$$

By duality, Theorem 3.2.6 implies Corollary 4.2.1.

Corollary 4.2.1. Let $\mathcal{K}$ be a 2 -category with finite limits and colimits, considered as equipped with the dual of its natural model 2-category structure. If $\mathcal{K}$ is complete, then for every small 2-category $\mathcal{A}$ the 2 -category $[\mathcal{A}, \mathcal{K}]$ admits the injective model structure. The injective model structure equips $[\mathcal{A}, \mathcal{K}]$ with a model 2 -category structure.

Proof. The 2-category $[\mathcal{A}, \mathcal{K}]$ can be identified with the 2 -category $\left[\mathcal{A}^{\mathrm{op}}, \mathcal{K}^{\mathrm{op}}\right]$. Considering the dual of the natural model structure $\mathcal{K}$ and the injective model structure on $[\mathcal{A}, \mathcal{K}]$ is the same as considering the natural model structure on $\mathcal{K}^{\text {op }}$ and the projective model structure on $\left[\mathcal{A}^{\mathrm{op}}, \mathcal{K}^{\mathrm{op}}\right]$. The latter exists by Theorem 3.2.6.

Duality can be used also to observe that the inclusion 2-functor in (10) admits not only a left 2 -adjoint but also a right 2 -adjoint:

$$
[\mathcal{A}, \mathcal{K}] \underset{\frac{1}{(\cdot)^{*}}}{\frac{\perp}{\longleftarrow}} \operatorname{Psd}[\mathcal{A}, \mathcal{K}]
$$

The unit of the 2-adjunction in (13) has components given by 2-natural transformations $r_{F}: F \rightarrow F^{*}$ that are pointwise injective equivalences, and so pointwise acyclic cofibrations in the injective model structure. The 2 -adjunction in (13) determines a 2 -monad on $[\mathcal{A}, \mathcal{K}]$ whose underlying 2-functor $R:[\mathcal{A}, \mathcal{K}] \rightarrow[\mathcal{A}, \mathcal{K}]$ is defined by letting $R F=I\left(F^{*}\right)$. Here, the unit of the 2-monad provides 2-functorial fibrant replacements for the injective model structure, since its components $r_{F}: F \rightarrow R F$ are acyclic cofibrations in the injective model structure, and each $R F$ is injectively fibrant. When $\mathcal{A}$ is a category and $\mathcal{K}$ is Cat, we will give explicit formulas for $R F$ in Section 6.4. We conclude this section by proving that the projective and the injective model structures are Quillen 2-equivalent.

Lemma 4.2.2. Let $\mathcal{A}$ be a small 2-category. Let $\mathcal{K}$ be a 2 -category with finite limits and colimits.

(i) If a 2-natural transformation has the right lifting property with respect to pointwise injective equivalences, then it is a pointwise isofibration. 
(ii) If a 2-natural transformation has the left lifting property with respect to surjective equivalences, then it is a pointwise isocofibration.

Proof. We prove $(i)$. Let $m: F \rightarrow G$ be a 2 -natural transformation and assume that it has the the right lifting property with respect to pointwise injective equivalences. By part $(i)$ of Lemma 4.1.2, to prove that it is a pointwise isofibration it is sufficient to prove that every component $m_{A}: F A \rightarrow G A$ has the right lifting property with respect to injective equivalences in $\mathcal{K}$. But this follows immediately since $m: F \rightarrow G$ has the right lifting property with respect to pointwise injective equivalences.

Proposition 4.2.3. The identity 2-functor induces a Quillen 2-equivalence between the projective and the injective model structure.

Proof. The claim follows from Lemma 4.2.2 by the same reasoning used in the proof of Proposition 4.1.3.

\section{Model Structures FOR HOMOtopy Limits}

5.1. Quillen 2-adjunctions in two variables. To study homotopy limits for 2categories, we use a straighforward 2-categorical analogue of the notion of a Quillen adjunction in two variables [14]. A 2-functor of the form $\Phi: \mathcal{K} \times \mathcal{L} \rightarrow \mathcal{M}$, where $\mathcal{K}, \mathcal{L}$, and $\mathcal{M}$ are 2-categories, will be referred to as a 2-functor in two variables. Given $\Phi: \mathcal{K} \times \mathcal{L} \rightarrow \mathcal{M}$, for $u: X \rightarrow Y$ in $\mathcal{K}$ and $v: V \rightarrow W$ in $\mathcal{L}$, we have the commutative diagram

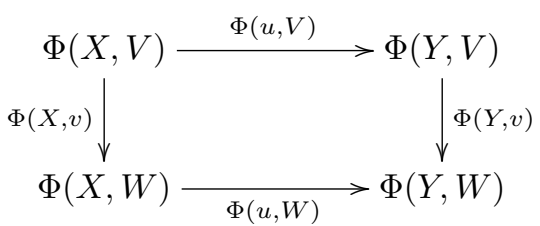

When $\mathcal{M}$ has pushouts, the commutativity of the diagram determines a canonical map

$$
\langle u, v\rangle: \Phi(X, W) \sqcup_{\Phi(X, V)} \Phi(Y, V) \rightarrow \Phi(Y, W) .
$$

When the underlying categories of $\mathcal{K}, \mathcal{L}, \mathcal{M}$ are equipped with model structures, we say that $\Phi: \mathcal{K} \times \mathcal{L} \rightarrow \mathcal{M}$ is a left Quillen 2-functor in two variables if $\Phi$ is cocontinuous in each variable, and if $u: X \rightarrow Y$ is a cofibration in $\mathcal{K}$ and $v: U \rightarrow V$ is a cofibration in $\mathcal{L}$, then $\langle u, v\rangle$ is a cofibration in $\mathcal{M}$, which is also a weak equivalence when either $u$ or $v$ is so. We say that $\Phi: \mathcal{K} \times \mathcal{L} \rightarrow \mathcal{M}$ is a right Quillen 2-functor in two variables if its dual $\Phi^{\mathrm{op}}: \mathcal{K}^{\mathrm{op}} \times \mathcal{L}^{\mathrm{op}} \rightarrow \mathcal{M}^{\mathrm{op}}$ is a left Quillen functor in two variables. Quillen 2-functors in two variables often arise in a special kind of situation, which is convenient to isolate. Recall that a 2-adjunction in two variables consists of 2 -functors

$$
\Phi: \mathcal{K} \times \mathcal{L} \rightarrow \mathcal{M}, \quad \Theta: \mathcal{L}^{\mathrm{op}} \times \mathcal{M} \rightarrow \mathcal{K}, \quad \Psi: \mathcal{K}^{\mathrm{op}} \times \mathcal{M} \rightarrow \mathcal{L},
$$

and 2-natural isomorphisms, for $X \in \mathcal{K}, Y \in \mathcal{L}$, and $Z \in \mathcal{M}$

$$
\mathcal{K}(X, \Theta(Y, Z)) \cong \mathcal{M}(\Phi(X, Y), Z) \cong \mathcal{L}(Y, \Psi(X, Z))
$$

In these circumstances, $\Phi$ is a left 2-adjoint in two variables, while $\Psi$ and $\Theta$ are right 2 -adjoints in two variables. This notion of two-variable adjunction for enriched categories has been studied in [11].

When the underlying categories of $\mathcal{K}, \mathcal{L}$ and $\mathcal{M}$ are equipped with model structures we have a Quillen 2-adjunction in two variables if the following equivalent conditions hold:

(i) $\Phi: \mathcal{K} \times \mathcal{L} \rightarrow \mathcal{M}$ is left Quillen 2-functor in two variables,

(ii) $\Theta: \mathcal{L}^{\text {op }} \times \mathcal{M} \rightarrow \mathcal{K}$ is right Quillen 2-functor in two variables,

(iii) $\Psi: \mathcal{K}^{\mathrm{op}} \times \mathcal{M} \rightarrow \mathcal{K}$ is right Quillen 2 -functor in two variables. 
The equivalence between these conditions is in [14, Lemma 4.2.2]. For example, when $\mathcal{K}$ is tensored and cotensored, the 2-adjunctions in (3) and (4) allow us to obtain a 2-adjunction in two variables involving the following 2-functors:

$$
\begin{array}{ll}
\Phi: \text { Cat } \times \mathcal{K} \rightarrow \mathcal{K}, & \Phi(A, X)={ }_{\text {def }} A \otimes X \\
\Theta: \mathcal{K}^{\text {op }} \times \mathcal{K} \rightarrow \text { Cat }, & \Theta(X, Y)={ }_{\text {def }} \mathcal{K}(X, Y) \\
\Psi: \text { Cat }^{\text {op }} \times \mathcal{K} \rightarrow \mathcal{K}, & \Psi(A, Y)={ }_{\text {def }} Y^{A} .
\end{array}
$$

Lemma 2.2.2 can then be rephrased as follows.

Lemma 5.1.1. Let $\mathcal{K}$ be a 2 -category with finite limits and colimits, whose underlying category is equipped with a model structure. When $\mathcal{K}$ is tensored and cotensored, $\mathcal{K}$ is a model 2-category if and only the following equivalent conditions hold.

(i) The tensor 2-functor is a left Quillen 2-functor in two variables.

(ii) The hom 2-functor is a right Quillen 2-functor in two variables.

(iii) The cotensor 2-functor is a right Quillen 2-functor in two variables.

5.2. Homotopical aspects of weighted limits. Existence of conical 2-limits in a 2 -category $\mathcal{K}$ can be expressed by saying that for every small 2 -category $\mathcal{A}$ we have a 2 -adjunction of the form

$$
\mathcal{K} \underset{<\frac{\Delta}{\lim }}{\frac{\Delta}{\mathcal{A}}}[\mathcal{K}] .
$$

The left 2-adjoint is the diagonal 2-functor, and the right 2-adjoint sends a diagram to its limit. When $\mathcal{K}$ is equipped with the dual of its natural model structure and $[\mathcal{A}, \mathcal{K}]$ with the injective model structure, we obtain a Quillen 2-adjunction, since the diagonal 2-functor clearly preserves weak equivalences and cofibrations. As an enriched category, however, $\mathcal{K}$ admits more general notions of limits, known as weighted limits [18, 27], whose homotopy-theoretic behaviour is less straighforward.

To recall weighted limits, we refer to 2 -functors $J: \mathcal{A} \rightarrow$ Cat as weights. Existence of weighed limits for a fixed diagram $F$ can be expressed as the existence of a 2-adjunction of the form

$$
\mathcal{K} \underset{\{-, F\}}{\frac{\mathcal{K}(\Delta(-), F)}{<}}[\mathcal{A}, \text { Cat }]^{\mathrm{op}}
$$

The left 2-adjoint sends $X \in \mathcal{K}$ into the weight $\mathcal{K}(X, F(-)): \mathcal{A} \rightarrow$ Cat, while the right 2-adjoint sends a weight $J: \mathcal{A} \rightarrow$ Cat to the $J$-weighted limit of $F$, denoted $\{J, F\}$ as usual [17]. Thus, the $J$-weighted limit of $F$ is characterized by 2-natural isomorphisms of form

$$
[\mathcal{A}, \mathbf{C a t}](J(-), \mathcal{K}(X, F(-))) \cong \mathcal{K}(X,\{J, F\}) .
$$

When $\mathcal{K}$ is tensored, for a fixed weight $J: \mathcal{A} \rightarrow$ Cat, existence of $J$-weighted limits can be expressed equivalently as the existence of a 2-adjunction of the form

$$
\mathcal{K} \underset{\{J,-\}}{\stackrel{J \otimes \Delta(-)}{<}}[\mathcal{A}, \mathcal{K}]
$$

The left 2-adjoint should be thought of as ' $J$-weighted diagonal': it sends $X \in \mathcal{K}$ into the diagram $J(-) \otimes X: \mathcal{A} \rightarrow \mathcal{K}$. The right 2-adjoint sends a diagram to its $J$-weighted limit. Therefore, we can characterize $\{J, F\}$ also by the existence of 2-natural isomorphism with components

$$
[\mathcal{A}, \mathcal{K}](J(-) \otimes X, F(-)) \cong \mathcal{K}(X,\{J, F\}) .
$$

It should be noted how the 2 -adjunction in (15) is analogous to that in (17). Writing $1: \mathcal{A} \rightarrow$ Cat for the weight with constant value the terminal category, it is 
immediate to see that weighted limits subsume conical limits $[17, \S 3.8]$, since the limit of a diagram $F$ can be viewed as the weighted limit by the existence of an isomorphism

$$
\lim F \cong\{\mathbf{1}, F\} .
$$

The map assigning to a weight and a diagram the corresponding weighted limit determines a 2-functor in two variables. By the 2-adjunctions in (16) and (17), the weighted limit 2 -functor is a right 2 -adjoint in two variables. It follows that there is a 2-adjunction in two variables involving the following 2-functors:

$$
\begin{array}{ll}
\Phi:[\mathcal{A}, \text { Cat }] \times \mathcal{K} \rightarrow[\mathcal{A}, \mathcal{K}], & \Phi(J, X)={ }_{\operatorname{def}} J(-) \otimes X \\
\Theta: \mathcal{K}^{\text {op }} \times[\mathcal{A}, \mathcal{K}] \rightarrow[\mathcal{A}, \text { Cat }], & \Theta(X, F)={ }_{\operatorname{def}} \mathcal{K}(X, F(-)) \\
\Psi:[\mathcal{A}, \text { Cat }]^{\text {op }} \times[\mathcal{A}, \mathcal{K}] \rightarrow \mathcal{K}, & \Psi(J, F)={ }_{\operatorname{def}}\{J, F\} .
\end{array}
$$

From now on, we assume that $\mathcal{K}$ is complete and cocomplete, so that we have both the projective and the injective model structure. We establish that there are two choices of model structures on functor 2-categories that allow us to regard the weighted limit 2-functor as a right Quillen 2-functor in two variables, and so the 2 -adjunction in two variables above as a Quillen 2 -adjunction in two variables. The first possibility is stated in Proposition 5.2.1.

Proposition 5.2.1. Considering both the 2-category of weights [ $\mathcal{A}, \mathbf{C a t}]$ and the 2 -category of diagrams $[\mathcal{A}, \mathcal{K}]$ as equipped with the projective model structure, the weighted limit 2-functor is a right Quillen 2-functor.

Proof. It suffices to verify that the 2-functor $\Theta$ defined in (19) is a right Quillen functor in two variables. This follows from the fact that the hom-category 2-functor is so, which holds by Lemma 5.1.1 and the fact that the natural model structure on $\mathcal{K}$ is a model 2 -structure.

As stated in Proposition 5.2.2, there exists a second choice of Quillen model structures that makes the weighted limit 2-functor into a right Quillen 2-functor in two variables.

Proposition 5.2.2. Considering both the 2-category of weights $[\mathcal{A}, \mathbf{C a t}]$ and the 2 -category of diagrams $[\mathcal{A}, \mathcal{K}]$ as equipped with the injective model structure, the weighted limit 2-functor is a right Quillen 2-functor in two variables.

Proof. It is sufficient to establish that the 2-functor $\Phi$ defined in (18) is a left Quillen 2 -functor in two variables. To prove this, it is sufficient to recall that the dual of the natural model structure is a model 2-structure and so, by Lemma 5.1.1, the tensor functor for $\mathcal{K}$ is a left Quillen 2-functor in two variables.

There are counterparts of these statements for weighted colimits, which we prefer to avoid stating to avoid repetition. To make the weighted colimit functor into a left Quillen 2-functor in two variables there are again two possible choices of model structures. The first involves the projective model structure on weights and the injective model structure on diagrams; the second involves the injective model structure on weights and the projective model structure on diagrams. The development in Section 6 has an evident analogue for colimits.

\section{6. Номотору Limits}

6.1. Relating pseudo-limits and homotopy limits. We wish to relate the notion of a pseudo-limit, as discussed in [3, 18, 27], to that of a homotopy limit. The notion of a conical pseudo-limits can be obtained from that of a conical limit by replacing 2-natural transformations with pseudo-natural transformations in the 2categories of diagrams involved in the definition of conical limits. More precisely, 
the existence of conical pseudo-limits in $\mathcal{K}$ is expressed by saying that for every small 2-category $\mathcal{A}$ we have a 2 -adjunction of form

$$
\mathcal{K} \underset{\operatorname{pslim}}{\stackrel{\Delta}{\gtrless}} \operatorname{Psd}[\mathcal{A}, \mathcal{K}] .
$$

Here the left 2-adjoint is obtained by composing the diagonal 2-functor in (17) with the inclusion 2-functor in (8). Note that, even if we are considering pseudo-natural transformations, we are still requiring that $(21)$ is a 2 -adjunction. Therefore, the pseudo-limit of a diagram $F$ is characterized by a 2-natural isomorphism

$$
\operatorname{Psd}[\mathcal{A}, \mathcal{K}](\Delta X, F) \cong \mathcal{K}(X, \operatorname{pslim} F) .
$$

Note that a pseudo-natural transformation $\Delta X \rightarrow F$ can be seen as a cone commuting up to coherent isomorphism.

The connection between pseudo-limits and homotopy limits follows from a sequence of observations. First, recall from [21, §2.4] that the localisation functor $\lambda:$ Cat $\rightarrow \mathrm{Ho}($ Cat $)$ preserves finite products. Hence, every 2 -category $\mathcal{K}$ has an associated $\mathrm{Ho}(\mathbf{C a t})$-category $\mathcal{K}_{\lambda}$ whose enrichement is defined by applying $\lambda$ to the hom-categories of $\mathcal{K}$. Secondly, let us recall that the homotopy limit functor is part of a $\mathrm{Ho}(\mathbf{C a t})$-enriched adjunction of the form

$$
\mathrm{Ho}(\mathcal{K}) \underset{\operatorname{holim}}{\frac{\operatorname{Ho}(\Delta)}{<}} \operatorname{Ho}([\mathcal{A}, \mathcal{K}]) .
$$

Such a $\mathrm{Ho}(\mathbf{C a t})$-adjunction exists since we may consider $\mathcal{K}$ as equipped with the dual of its natural model structure and $[\mathcal{A}, \mathcal{K}]$ as equipped with the corresponding injective model structure. Thirdy, we may observe that $\operatorname{Ho}(\mathcal{K})$ and $\operatorname{Ho}([\mathcal{A}, \mathcal{K}])$ can be identified with the $\operatorname{Ho}(\mathbf{C a t})$-categories $\mathcal{K}_{\lambda}$ and $\operatorname{Psd}[\mathcal{A}, \mathcal{K}]_{\lambda}$ associated to the 2categories $\mathcal{K}$ and $\operatorname{Psd}[\mathcal{A}, \mathcal{K}]$, respectively. This remark, inspired by an analogous observation in $[21, \S 4.14]$, follows by a direct calculation that involves instaciating the general definition of the $\mathrm{Ho}(\mathbf{C a t})$-enriched category associated to a model 2category. As a consequence of this, the $\mathrm{Ho}(\mathbf{C a t})$-adjunction in (22) is nothing but the $\mathrm{Ho}(\mathbf{C a t})$-adjunction associated to the 2-adjunction in (21) by the localisation functor $\lambda$ : Cat $\rightarrow \mathrm{Ho}(\mathbf{C a t})$.

This reasoning implies that a pseudo-limit, when regarded as an object of the homotopy $\mathrm{Ho}$ (Cat)-category, is a homotopy limit. An analogous statement holds for weighted pseudo-limits, which can be defined by replacing 2-natural transformations with pseudo-natural transformations not only in 2-categories of diagrams, but also in 2-categories of weights. In the following, we write $\{J, F\}_{p}$ for the $J$-weighted pseudo-limit of $F$.

6.2. Two resolutions. Proposition 5.2.1 and Proposition 5.2.2 allow us to apply the general theory of model $\mathcal{V}$-categories $[9,14]$ and conclude the existence of the total derived $\mathrm{Ho}(\mathbf{C a t})$-enriched functor of the weighted limit 2-functor. We refer to it as the weighted homotopy limit functor. We wish to show how the two different ways of expressing homotopy weighted limits as weighted limits suggested by Proposition 5.2.1 and Proposition 5.2.2 correspond exactly to two ways of expressing weighted pseudo-limits as weighted limits.

If we consider both the 2-category of diagrams and the 2-category of weights as equipped with the projective model structure, Proposition 5.2.1 leads us to define the total right derived functor of the weighted limit 2-functor by letting, for $J$ : $\mathcal{A} \rightarrow$ Cat and $F: \mathcal{A} \rightarrow \mathcal{K}$

$$
\{J, F\}^{\mathrm{R}}={ }_{\text {def }}\{Q J, F\} .
$$

Here $Q J$ denotes the cofibrant replacement of the weight $J$ with respect to the projective model structure, as in Section 3.2. Note that it is not necessary to 
replace $F$ since every diagram is projectively fibrant. This formula is closely related to a well-known result showing that the existence of weighted limits implies the existence of weighted pseudo-limits [3, 18]. Indeed, using the left 2-adjoint in (10), we have the sequence of 2-natural isomorphisms

$$
\begin{aligned}
\mathcal{K}\left(X,\{J, F\}_{p}\right) & \cong \operatorname{Psd}[\mathcal{A}, \mathbf{C a t}](J, \mathcal{K}(X, F(-))) \\
& \cong[\mathcal{A}, \mathbf{C a t}](Q J, \mathcal{K}(X, F(-))) \\
& \cong \mathcal{K}(X,\{Q J, F\})
\end{aligned}
$$

The Yoneda lemma for 2-categories implies the existence of an isomorphism

$$
\{J, F\}_{p} \cong\{Q J, F\} .
$$

There is a different, but equivalent, definition for the total right derived functor of the weighted limit 2-functor, which follows from Proposition 5.2.2. If we consider the weighted limit 2-functor as a Quillen 2-functor in two variables with respect to the injective model structures, its total right derived $\mathrm{Ho}(\mathbf{C a t})$-enriched functor can be defined by letting

$$
\{J, F\}^{\mathrm{R}}={ }_{\text {def }}\{J, R F\} .
$$

Here $R F: \mathcal{A} \rightarrow \mathcal{K}$ denotes the fibrant replacement of a diagram $F: \mathcal{A} \rightarrow \mathcal{K}$ with respect to the injective model structure, as in Section 4.2. Note that we do not need to make any replacement for $J$, since any weight is injectively cofibrant. This corresponds to a different way of expressing pseudo-limits in terms of weighted limits, which does not seem to appear in the existing literature. Assuming that $\mathcal{K}$ has tensors, and using the right 2-adjoint in (13), we have the sequence of 2-natural isomorphisms

$$
\begin{aligned}
\mathcal{K}\left(X,\{J, F\}_{p}\right) & \cong \operatorname{Psd}[\mathcal{A}, \mathcal{K}](J(-) \otimes X, F(-)) \\
& \cong[\mathcal{A}, \mathcal{K}](J(-) \otimes X, R F(-))) \\
& \cong \mathcal{K}(X,\{J, R F\})
\end{aligned}
$$

Hence, we conclude as before that there is an isomorphism

$$
\{J, F\}_{p} \cong\{J, R F\} .
$$

6.3. Homotopy limits. The two choices of model structures making the weighted limit functor into a right Quillen 2-functor can be used also in the computation of homotopy limits. Let us now consider $\mathcal{K}$ as being equipped with its natural model 2 -structure. We can therefore define the homotopy limit of a diagram $F$ by letting

$$
\operatorname{holim} F={ }_{\operatorname{def}}\{\mathbf{1}, F\}^{\mathrm{R}}
$$

A first way to compute the homotopy limit is to apply the formula in (23) and obtain

$$
\operatorname{holim} F=\{Q \mathbf{1}, F\}
$$

This is in fact a consequence of a well-known formula $[3,18,27]$ expressing the pseudo-limit of a diagram $F: \mathcal{A} \rightarrow \mathcal{K}$ as the weighted limit $\{Q 1, F\}$. The formula has at least two noteworthy aspects. First, it involves only the projective model structures. Hence, in the context of model 2-categories, homotopy limit 2-functors can be defined without injective model structures. Secondly, $Q 1$ is the cofibrant replacement of the constant weight $\mathbf{1}: \mathcal{A} \rightarrow$ Cat with respect to the projective model structure on $[\mathcal{A}, \mathbf{C a t}]$. Even if cofibrant replacements with respect to projective model structures are generally rather involved, the very simple nature of the weight simplifies the task considerably. If we apply the formula in (25), instead, we obtain

$$
\operatorname{holim} F=\lim R F .
$$


This is the simplest homotopy-theoretic formula for the homotopy limit of a diagram, which involves considering the injective model structure on $[\mathcal{A}, \mathcal{K}]$ and computing the right derived functor of the limit functor. Remarkably, this corresponds to a way of expressing pseudo-limits in terms of conical 2-limits and of the 2adjunction in (13) which does not seem to appear in the existing literature on 2-categories. Indeed, using the right 2-adjoint in (13), we get

$$
\begin{aligned}
\mathcal{K}(X, \operatorname{pslim} F) & =\operatorname{Psd}[\mathcal{A}, \mathcal{K}](\Delta X, F) \\
& =[\mathcal{A}, \mathcal{K}](\Delta X, R F) \\
& \cong \mathcal{K}(X, \lim R F) .
\end{aligned}
$$

Hence, we obtain an isomorphism

$$
\operatorname{pslim} F \cong \lim R F .
$$

This isomorphism, which is a special case of (26), expresses pseudo-limits in terms of conical 2-limits and the 2 -adjunction in (13). The assumption that $\mathcal{K}$ is complete is necessary to do so. Indeed, as discussed in [3, §2], the full sub-2-category of Cat whose objects are the categories with at most one object has all conical limits, but not all pseudo-limits.

6.4. Some formulas. When $\mathcal{A}$ is an ordinary category and $\mathcal{K}$ is Cat, it is possible to provide some explicit formulas for the 2-monad and 2-comonad providing the fibrant replacement and cofibrant replacement in the injective and projective model structures, respectively, discussed in Section 3.2 and Section 4.2. For notational convenience, we prefer to consider contravariant functors. The right 2-adjoint admits a simple description, which is determined by the Yoneda Lemma for 2-categories. For $A \in \mathcal{A}$, an object of $F^{*} A$ can be identified with a 2-natural transformation $\mathbf{y}(A) \rightarrow F^{*}$, where $\mathbf{y}(A): \mathcal{A}^{\text {op }} \rightarrow$ Cat denotes the Yoneda embedding of $A$. By 2-adjointness, this 2-natural transformation should correspond to a pseudo-natural transformation $\mathbf{y}(A) \rightarrow F$. Hence, we are led to define the 2-monad for fibrant replacements as

$$
R F(A)=_{\text {def }} \operatorname{Psd}[\mathcal{A}, \mathbf{C a t}](\mathbf{y}(A), F) .
$$

The right-hand side can be described equivalently in terms of cartesian sections of Grothendieck fibrations, as in [10, §I.2.4.4.1].

A formula for the 2-comonad follows by a direct application of the results in $[10$, $\S$ I.2.4]. We write $\operatorname{Fib}(\mathcal{A})$ for the 2-category of Grothendieck fibrations over $\mathcal{A}$, cartesian functors, and fibred natural transformations [10, §I.1.8]. The familiar Grothendieck construction $[12, \S$ VI.8 $]$ provides a 2 -functor Tot : $\left[\mathcal{A}^{\text {op }}, \mathbf{C a t}\right] \rightarrow$ $\operatorname{Fib}(\mathcal{A})$ mapping a functor $F: \mathcal{A}^{\mathrm{op}} \rightarrow$ Cat into a split Grothendieck fibration Tot $F \rightarrow \mathcal{A}$. By $[10, \S \mathrm{I} .2 .4 .3]$ this 2 -functor has a left 2-adjoint $L: \operatorname{Fib}(\mathcal{A}) \rightarrow$ $\left[\mathcal{A}^{\text {op }}, \mathbf{C a t}\right]$. Hence we obtain the following isomorphisms

$$
\left[\mathcal{A}^{\mathrm{op}}, \mathbf{C a t}\right](L(\operatorname{Tot} F), G) \cong \mathbf{F i b}(\mathcal{A})(\operatorname{Tot} F, \operatorname{Tot} G) \cong \operatorname{Psd}\left[\mathcal{A}^{\mathrm{op}}, \mathbf{C a t}\right](F, G)
$$

The first isomorphisms follows by the 2-adjointness $L \dashv$ Tot, while the second follows by the identification between pseudo-natural transformations $F \rightarrow G$ and cartesian functors $\operatorname{Tot} F \rightarrow \operatorname{Tot} G$. We can therefore define the 2-comonad for cofibrant replacements by letting

$$
Q F={ }_{\operatorname{def}} L(\operatorname{Tot} F) .
$$

We wish to unwind this definition. For $A \in \mathcal{A}$, let us write $\mathcal{A}^{\backslash A}$ for the category whose objects are the arrows in $\mathcal{A}$ with domain $A$, and with maps the evident commuting triangles. There is then a canonical functor $\mathcal{A} \backslash A \rightarrow \mathcal{A}$ mapping an arrow into its codomain, and we write $\operatorname{Tot}(F) \backslash A \rightarrow \mathcal{A} \backslash A$ for the Grothendieck 
fibration obtained by pulling back the Grothendieck fibration $\operatorname{Tot} F \rightarrow \mathcal{A}$ along it. By the definitions in $[10, \S$ I.2.4.3] we obtain

$$
Q F(A)=\underline{\operatorname{Lim}}\left(\operatorname{Tot}(F)^{\backslash A} / \mathcal{A}^{\backslash A}\right),
$$

The right-hand side denotes the category obtained by localizing the category $\operatorname{Tot}(F) \backslash A$ with respect to the set of its cartesian morphisms, with universal properties as in $[2$, $\S \mathrm{VI} .6]$.

Acknowledgements. I would like to thank André Joyal for several helpful discussions. I am also grateful to Richard Garner, Claudio Hermida, Martin Hyland, Steve Lack, Michael Mandell, John Power, Alexandru Stanculescu, and Myles Tierney for useful conversations. Part of the material in the paper was presented at the workshop "Identity Types: Topological and Categorical Structure" at the University of Uppsala. I would like to thank its organizers for the interesting conference, and André Joyal and Christophe Reutenauer for supporting my participation. I am also grateful to the participants of Category Theory seminar at McGill University and of the CRM seminars, where this material was presented, for their comments.

The research described here was developed during a postdoctoral research fellowship at the Université du Québec à Montréal. I would like to thank the Laboratoire de Combinatorie et Informatique Mathématique for the support. This paper will appear in the Mathematical Proceedings of the Cambridge Philosophical Society. I am grateful to the editors for granting permission to publish it in preprint form, and the anonymous referee for providing useful comments. The final version of this paper was prepared while holding a research fellowship at the Centre de Recerca Matemàtica (CRM) in Barcelona. I am grateful to the CRM for its support and hospitality, and to the University of Leicester for granting me a research leave for the academic year 2007/08.

\section{Appendix A. Quillen model Categories}

A Quillen model structure on a category $\mathcal{K}$ consists of three classes of maps $\mathcal{W}, \mathcal{F}, \mathcal{C}$, whose elements are called respectively weak equivalences, fibrations, and cofibrations, satisfying the axioms (Q1)-(Q4) stated below. In stating these axioms, we refer to maps in $\mathcal{W} \cap \mathcal{F}$ as acyclic fibrations and to maps in $\mathcal{W} \cap \mathcal{C}$ as acyclic cofibrations.

(Q1): For every commutative diagram of the form

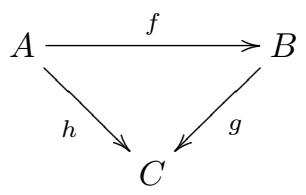

if two out of $f, g$, and $h$ are weak equivalences, then so is the third.

(Q2): For any commutative diagram of the form

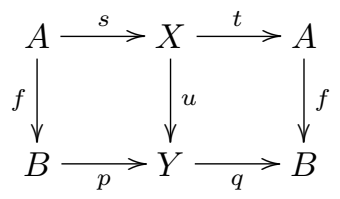

such that $t s=1_{A}$ and $q p=1_{B}$, if $u$ is a weak equivalence, fibration, or cofibration, then so is $f$. 
(Q3): For any commutative square of the form

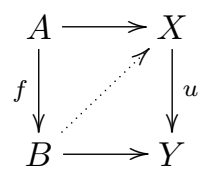

such that $f$ is a cofibration and $u$ is a fibration, if either of $f$ or $u$ is a weak equivalence, then there exists a dotted filler.

(Q4): Every map in $\mathcal{K}$ can be factored both as an acyclic cofibration followed by a fibration and as a cofibration followed by an acyclic fibration.

A Quillen model category consists of a finitely complete and cocomplete category equipped with a Quillen model structure.

\section{REFERENCES}

[1] D. W. Anderson. Fibrations and geometric realizations. Bulletin of the American Mathematical Society, 84(5):765-788, 1978 .

[2] M. Artin, A. Grothendieck, and J. L. Verdier. Théorie des Topos et Cohomologie Etale des Schémas (SGA 4), volume 270 of Lecture Notes in Mathematics. Springer, 1972.

[3] G. J. Bird, G. M. Kelly, A. J. Power, and R. H. Street. Flexible limits for 2-categories. Journal of Pure and Applied Algebra, 61:1 - 27, 1989.

[4] R. Blackwell, G.M. Kelly, and A. J. Power. Two-dimensional monad theory. Journal of Pure and Applied Algebra, 59:1 - 41, 1989.

[5] A.K. Bousfield and D.M. Kan. Homotopy limits, completions, and localizations, volume 304 of Lecture Notes in Mathematics. Springer, 1972.

[6] W. Chachólski and J. Scherer. Homotopy theory of diagrams. Memoirs of the American Mathematical Society, 155(736), 2002

[7] W. G. Dwyer, P. S. Hirschhorn, D. M. Kan, and J. H. Smith. Homotopy limit functors on model categories and homotopical categories, volume 113 of Mathematical Surveys and Monographs. American Mathematical Society, 2004.

[8] R. Garner. Cofibrantly generated natural weak factorisation systems. ArXiv:math/0702290, 2007.

[9] L. Gaunce Lewis, Jr. and M. A. Mandell. Modules in monoidal model categories. Journal of Pure and Applied Algebra, 210:395-421, 2007.

[10] J. Giraud. Cohomologie non abélienne. Springer, 1971.

[11] J. Gray. Closed categories, lax limits, and homotopy limits. J. Pure Applied Algebra, 19:127158,1980

[12] A. Grothendieck. Revêtements étales et groupe fondamental (SGA 1), volume 224 of Lecture Notes in Mathematics. Springer, 1971.

[13] P. Hirschhorn. Model categories and their localizations, volume 99 of Mathematical Surveys and Monographs. American Mathematical Society, 2002.

[14] M. Hovey. Model categories, volume 63 of Mathematical Surveys and Monographs. American Mathematical Society, 1998.

[15] A. Joyal and M. Tierney. Strong stacks and classifying spaces. In Category Theory (Como 1990), volume 1488 of Lecture Notes in Mathematics, pages 213-236. Springer, 1991.

[16] G. M. Kelly. Doctrinal adjunction. In Kelly and Street [19], pages 257-280.

[17] G. M. Kelly. Basic concepts of enriched category theory, volume 64 of London Mathematical Society Lecture Note. Cambridge University Press, 1982. Available online in the Reprints in Theory and Applications of Categories.

[18] G. M. Kelly. Elementary observations on 2-categorical limits. Bulletin of the Australian Mathematical Society, 39(2):301 - 317, 1989.

[19] G. M. Kelly and R. H. Street, editors. Category Seminar (Proc. Sem. Sydney 1972/1973), volume 420 of Lecture Notes in Mathematics. Springer, 1974.

[20] G. M. Kelly and R.H. Street. Review of the elements of 2-categories. In Kelly and Street [19], pages $75-103$

[21] S. Lack. Homotopy-theoretic aspects of 2-monads. ArXiv:math/0607646, 2006. To appear in the Journal of Homotopy and Related Structures.

[22] J. P. May. The geometry of iterated loop spaces, volume 271 of Lecture Notes in Mathematics. Springer, 1972.

[23] J. Power and E. Robinson. A characterisation of pie limits. Mathematical Proceedings of the Cambridge Philosophical Society, 110(1):33-47, 1991.

[24] C. Rezk. A model category for categories. Available from the author's web page, 1996. 
[25] S. Schwede and B. Shipley. Algebras and modules in monoidal model categories. Proceedings of the London Mathematical Society, 80(2):491-511, 2000.

[26] M. Shulman. Homotopy limits and colimits and enriched homotopy theory. ArXiv:math/0610194, 2006.

[27] R. Street. Limits indexed by category-valued 2-functors. Journal of Pure and Applied Algebra, 8:149-181, 1976.

[28] R. W. Thomason. Cat as a closed model category. Cahiers de Topologie et Géometrie Différentielle, XXI(3):305-324, 1980.

Department of Computer Science, University of Leicester, University Road, LeicesTER LE1 7RH, ENGLAND

E-mail address: nicola.gambino@gmail.com 